\title{
A MATHEMATICAL MODEL FOR INTRACELLULAR EFFECTS OF TOXINS ON DNA ADDUCTION AND REPAIR
}

- D. P. GAVER and P. A. JACOBS

Department of Operations Research, Naval Postgraduate School, Monterey, CA 93943, U.S.A.

\section{- R. L. CARPENTER}

Naval Medical Research Institute, Wright Patterson AFB, Dayton, OH 45433, U.S.A.

\author{
J. G. BURKHART \\ Environmental Toxicology Program, \\ NIEHS, \\ Research Triangle Park, NC 27709, U.S.A.
}

\begin{abstract}
The processes by which certain classes of toxic compounds or their metabolites may react with DNA to alter the genetic information contained in subsequent generations of cells or organisms are a major component of hazard associated with exposure to chemicals in the environment. Many classes of chemicals may form DNA adducts and there may or may not be a defined mechanism to remove a particular adduct from DNA independent of replication. Many compounds and metabolites that bind DNA also readily bind existing proteins; some classes of toxins and DNA adducts have the capacity to inactivate a repair enzyme and divert the repair process competitively. This paper formulates an intracellular dynamic model for one aspect of the action of toxins that form DNA adducts, recognizing a capacity for removal of those adducts by a repair enzyme combined with reaction of the toxin and/or the DNA adduct to inactivate the repair enzyme. This particular model illustrates the possible saturation of repair enzyme capacity by the toxin dosage and shows that bistable behavior can occur, with the potential to induce abrupt shifts away from steady-state equilibria. The model suggests that bistable behavior, dose and variation between individuals or tissues may combine under certain conditions to amplify the biological effect of dose observed as DNA adduction and its consequences as mutation. A model recognizing stochastic phenomena also indicates that variation in within-cell toxin concentration may promote jumps between stable equilibria. (C) 1997 Society for Mathematical Biology
\end{abstract}

1. Introduction. This paper formulates a simple generic mathematical model for one aspect of the action of toxins that bind to DNA, react to interact with a repair enzyme and are removed within cells. It is an intracellular dynamic model that describes a process of formation of DNA adducts when there is a capacity for removal of those adducts from the DNA by a repair enzyme. Many compounds and metabolities that bind 
DNA also readily bind existing proteins; some classes of toxins and DNA adducts may inactivate the repair enzyme and subvert the repair process competitively (Chae et al., 1994; Lijinsky et al., 1994; Mineura et al., 1994). This particular model illustrates the possible saturation of repair enzyme capacity by the toxin dosage and shows that bistable behavior can sometimes occur, inducing abrupt shifts between two possible steady-state equilibria. The bistable behavior, dose and variation between individuals or tissues may combine under certain conditions to amplify the biological effect of dose, observed as DNA adduction. Models recognizing stochastic phenomena are also discussed; stochastic variation in within-cell toxin concentration can promote jumps across stable equilibria, as shown later.

The correct replication of a cell and its DNA is essential to the differentiation and maintenance of populations of cells within tissues and ultimately within host individuals, e.g. those making up a human population. Individual diploid human cells contain approximately $6 \times 10^{9}$ DNA base pairs comprising about $10^{6}$ genes (Burkhart, 1995). Risk assessment technology and practice benefit from insight provided by biologically based mechanistic models. The adduct/removal model presented here focuses only on one part of a complex intracellular process. Ultimately, models are required that describe the dynamics and effects of dose, cell surface recognition/transport, intracellular DNA/protein interaction, repair, detoxification and clearance. At each step there is potential for a cell to control, modify or possibly succumb to exogenously originating threats from toxic chemicals or their metabolities. Modification may also arise from interaction within cell populations through the action of exogenous signaling compounds. These interactions are to be the subjects of subsequent investigation.

2. Intracellular Model of Damage and Repair. Chemical or radiological environmental stress agents may induce DNA damage as a lesion, such as a single or double DNA strand break, or oxidative- and hydroxyl radical-induced changes in structure. Damage also takes the form of an adduct, where reactive chemical groups may chemically react with the purine/pyrimidine structures of DNA to disrupt hydrogen bond pairing or the action of polymerases (Singer, 1985; for review, see Board on Environmental Studies and Toxicology, 1989). The adducted DNA, if not repaired by removal of the alkyl or aryl group may result in an altered DNA sequence (mutation) that is passed on to subsequent daughter cells. Depending on the site (gene) that is mutated, many biological outcomes are conceivable (Michaelson, 1993). If the event contributes to abnormal cell cycle regulation, such that the cell becomes a candidate for entry into the carcinogenic process, the interaction is of particular interest.

The most frequently described adducts are methylated or ethylated nucleotides generated by direct alkylating agents (Bronstein et al., 1992; Pegg, 1990). These provide examples of the importance of the process in 
terms of mutation and of the potential for biological variation between tissues and individual to have profound effects on the mutagenic/carcinogenic outcome (Fox and Margison, 1988; Bronstein et al., 1991). Many toxic compounds are also likely to bind intracellular proteins, including those involved in the removal of an adduct from the DNA. There also may be a "suicide" reaction wherein the repair enzyme becomes inactivated and is thus removed from the population of molecules available for subsequent use by the cell in DNA repair (Hora et al., 1983; Pegg and Byers, 1992).

DNA adduction is one case among several possibilities, but it serves to motivate formulation of the generic mathematical models to follow. These are simplified extensively, but do capture some of the essentials of an adduct formation-repair/removal process in the presence of a toxic chemical and repair enzyme. Examination of the result of the model then suggests questions concerning system dynamics that are relevant to other intra- and extracellular processes and to risk assessment. These must ultimately be answered by appropriate experiments.

2.1. Deterministic mathematical model. Suppose the cell in question is viewed at time $t$, where $t$ is measured from cell division. To be specific let it be a stem cell, possibly in the spleen (cf. Alberts et al., 1994), so during time $t$ the cell may have produced a number of daughter cells, while itself remaining alive.

We initially write a deterministic kinetic model to represent the system, utilize the following notation: $A(t)$ is the (mean) number of adducts present on the DNA of the cell, $R(t)$ is the (mean) number or concentration of repair enzyme, e.g. alkyl DNA transferase, present in the cell interior and $T(t)$ is the concentration of the toxic chemical or activated metabolite thereof simultaneously present in the cell, all at time $t$. These quantities are stipulated to satisfy the following differential equations:

$$
\begin{aligned}
\frac{d A(t)}{d t}= & \underbrace{\lambda_{0}+\lambda_{1} T(t)}_{\text {adduct formation }}-\underbrace{\mu_{R A} R(t) A(t)}_{\begin{array}{c}
\text { adduct removal/repair } \\
\text { by enzyme } \\
\text { (DNA transferase) }
\end{array}}-\underbrace{\delta_{A M} A(t)}_{\begin{array}{c}
\text { non-enzymatic } \\
\text { adduct removal }
\end{array}}, \\
\frac{d T(t)}{d t}= & \underbrace{\tau_{C}(t)}_{\begin{array}{c}
\text { toxin input } \\
\text { to cell interior }
\end{array}}-\underbrace{\delta_{T} T(t)}_{\begin{array}{c}
\text { toxin removal; } \\
\text { other agents }
\end{array}}-\underbrace{\mu_{R T} R(t) T(t)}_{\begin{array}{c}
\text { toxin removal; } \\
\text { binds with }
\end{array}}, \\
\frac{d R(t)}{d t}= & \underbrace{\beta(\bar{R}-R(t))}_{\begin{array}{c}
\text { (D)icide repair enzyme" } \\
\text { enzyme creation }
\end{array}}-\underbrace{\delta_{R} R(t)}_{\begin{array}{c}
\text { enzymerase) } \\
\text { life-in-cell effect } \\
\text { or "turnover" }
\end{array}} \underbrace{\delta_{\text {by adduct repair }}}_{\text {enzyme removal }}
\end{aligned}
$$


These equations exhibit the possible double amplification effect of a toxin on adduct formation: first, the toxin contributes to the rate of adduct formation in accordance with rate parameter $\lambda_{1}$ (see (2.1)); second, it is in competition for the repair enzyme, thus depleting the enzyme's level, in accordance with the rate parameter $\mu_{R T}$ (see (2.2)). The expression $\delta_{T} T(t)$ in (2.2) includes loss of toxin as a result first of its removal during adduct formation as in (2.1); second, because of normal cellular processes such as detoxification or binding to DNA and proteins. Both a cell DNA adduct-enzyme product and a toxin-enzyme product may be induced; one example among many is the binding of benzylguanine to the alkyl DNA transferase (Chae et al., 1994).

The specific enzyme creation term incorporated in (2.3) is biologically plausible in that, without toxins and adducts, it expresses the long-run steady-state enzyme concentration as $R(\infty)=\bar{R} \beta /\left(\beta+\delta_{R}\right)$, relaxing exponentially to that level if perturbed; the rate of creation never exceeds $\beta^{*}=\beta R$. An alternative could be of the form $g(R(t))=\beta^{*} /\left[1+\kappa(R(t))^{p}\right]$, for $\kappa, p$ positive; i.e. a Michaelis-Menton $(p=1)$ or Hill $(p>1)$ function. Of course all parameters are, realistically, susceptible to individual betweencell variation, and the actual dependence on toxin level might well depend on past history in a manner more complex than is represented by (2.1)-(2.3). For the present all parameters will be treated as constants. One aim of our modeling is to expose unexpected effects and sensitivities, and some such are revealed even for the present simplified setup. All assumptions made are hypothetical artifacts of the model until verified empirically, but may serve to suggest particular experiments.

3. Steady-State Solution. Suppose all parameters and the rate of toxin input $\tau_{C}$ are temporarily assumed to be constants. If there is a long-run steady-state solution for $(A(t), T(t), R(t))$, then it satisfies the non-linear algebraic equations obtained by putting the derivatives equal to zero. In the present case steady-state solutions are obtained by solving the derivative $=$ zero (2.1) for $A$ in terms of $R,(2.2)$ for $T$ in terms of $R$ and finally putting these into (2.3). The result is

$$
\begin{aligned}
\beta \bar{R}= & \left(\beta+\delta_{R}\right) R+\mu_{R A}\left(\frac{\lambda_{0}}{\delta_{A M}+\mu_{R A} R}+\frac{\lambda_{1} \tau_{C}}{\left(\delta_{T}+\mu_{R T} R\right)\left(\delta_{A M}+\mu_{R A} R\right)}\right) R \\
& +\frac{\mu_{R T} \tau_{C} R}{\delta_{T}+\mu_{R T} R} .
\end{aligned}
$$

After multiplying out by the denominator there results a cubic equation for the fixed points which are the appropriate solutions of (3.1). Although parametric solutions can be found for (3.1) it will not be easy to extract 
general qualitative information from them; see the Appendix. A straightforward alternative is to introduce hypothetical biologically plausible parameters and to solve the differential equations (2.1), (2.2) and (2.3) numerically; this has been done in some trial cases.

Alternatively, important qualitative information can be obtained from the formula (3.1), written as follows:

$$
\begin{aligned}
\beta(\bar{R}-R)= & \delta_{R} R+\left(\frac{\lambda_{0} \mu_{R A}}{\delta_{A M}+\mu_{R A} R}+\frac{\tau_{C} \mu_{R T}}{\delta_{T}+\mu_{R T} R}\right) R \\
& +\frac{\lambda_{1} \tau_{C} \mu_{R A} R}{\left(\delta_{T}+\mu_{R T} R\right)\left(\delta_{A M}+\mu_{R A} R\right)}
\end{aligned}
$$

or

$$
l(R)=r(R) \equiv r_{1}(R)+r_{2}(R)+r_{3}(R) .
$$

Graph the left-hand side of the above expression as a function of $R$, written $l(R)=\beta(\bar{R}-R)$ vs the right-hand side, $r(R)=r_{1}(R)+r_{2}(R)+$ $r_{3}(R)$, where

$$
\begin{aligned}
& r_{1}(R)=\delta_{R} R \\
& r_{2}(R)=\left(\frac{\lambda_{0} \mu_{R A}}{\delta_{A M}+\mu_{R A} R}+\frac{\tau_{C} \mu_{R T}}{\delta_{T}+\mu_{R T} R}\right) R \\
& r_{3}(R)=\left(\frac{\lambda_{1} \tau_{C} \mu_{R A} R}{\left(\delta_{T}+\mu_{R T} R\right)\left(\delta_{A M}+\mu_{R A} R\right)}\right)
\end{aligned}
$$

Points of crossing are candidate solutions. Note the possible qualitative configuration of the above components as shown in Fig. 1. Now if the right-hand side components are summed, the qualitative possibilities may emerge (depending upon parameter values) as shown in Fig. 2. The curve

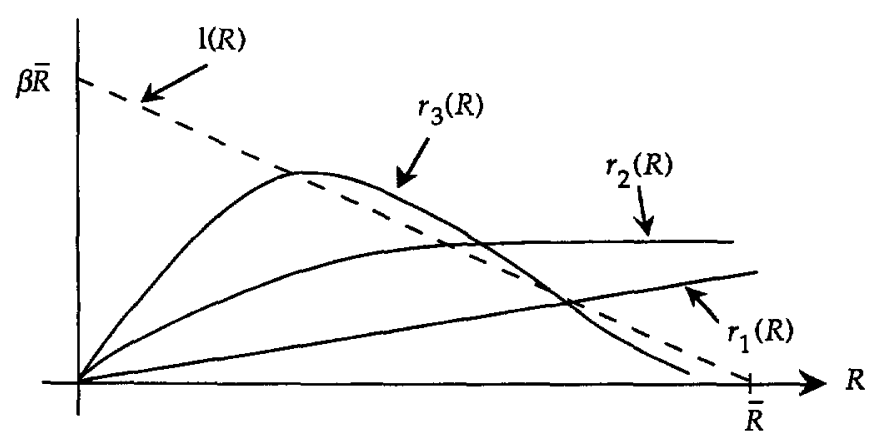

Figure 1. Terms of cubic equation. 


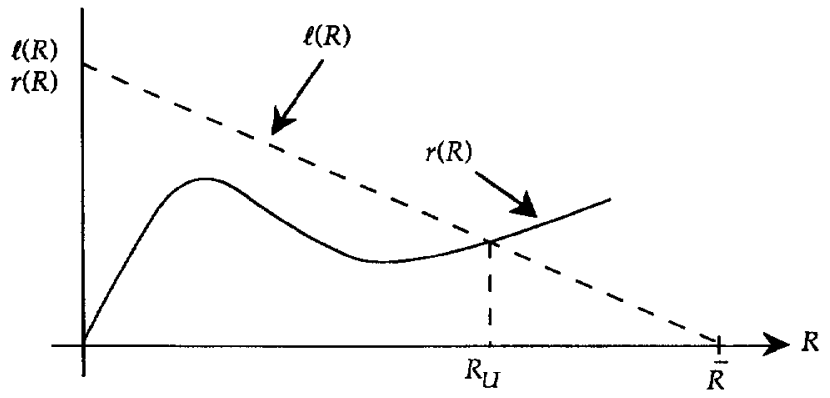

(a)

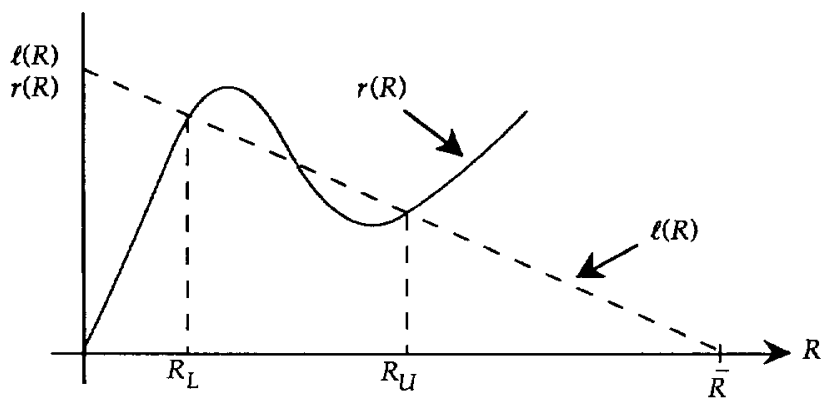

(b)

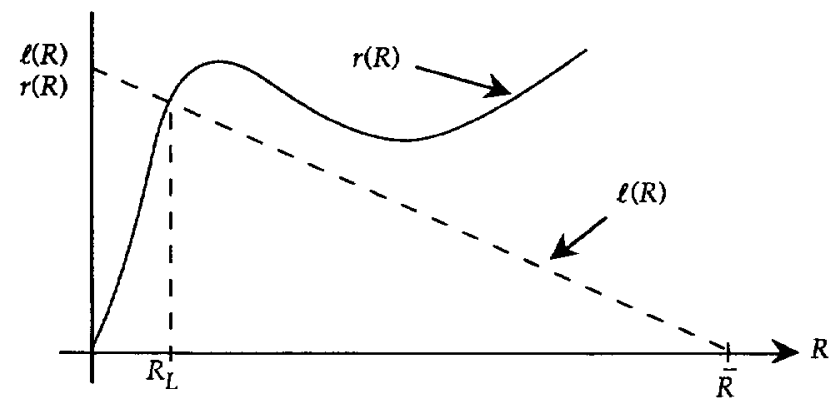

(c)

Figure 2. Possibilities of real solutions of cubic equation.

crossings, at values $R_{U}$ and $R_{L}$ in Fig. 2, actually represent local equilibrium points; cf. Beltrami (1987) and Strogatz (1994). It is conjectured (and has been verified numerically in trial cases by examination of eigenvalues for the linearized system, using MATLAB) that in case the oscillating behavior of $r(R)$ occurs as shown, the value $R_{U}$ in Fig. $2 \mathrm{a}, R_{U}$ and $R_{L}$ in Fig. $2 \mathrm{~b}$ and $R_{L}$ in Fig. $2 \mathrm{c}$ are all local stable points near which $R(t)$ will tend to reside as $t$ increases, provided the starting level is near one of these values. If toxin dosage to the cell $\tau_{C}$ is low, this is consistent with a relatively high level of enzyme; see formulas $r_{2}(R)$ and $r_{3}(R)$ in (3.1a) and Fig. $2 \mathrm{a}$. If $\tau_{C}$ is high, this is consistent with relatively low ambient enzyme 
(Fig. 2c). The intermediate equilibrium point in Fig. $2 b$ is presumably always locally unstable. Figure $2 \mathrm{~b}$ suggests the existence of two such stable points: $R_{U}$ and $R_{L}$. If the enzyme level starts near one of these, it tends to reside nearby, but if a disturbance (the origin of which is not modeled here) occurs, the enzyme level may abruptly shift, e.g. from $R_{U}$ to $R_{L}$, consistent with a high level of ambient toxin and a relatively high formation rate for adducts. Switches back and forth may occur. Such behavior has been called bistable. The conditions described tend to be associated with a relatively high rate of fixation by mutation when $R(t)$ is near $R_{L}$, with subsequent damaging effects. However, other protective behavior such as apoptosis (programmed cell death) may be stimulated to inhibit overall adduct fixation and tumor production. Apoptosis is not modeled here.

Graphical analysis shows that invocation of a Michaelis-Menton or Hill expression for enzyme creation may produce results that are qualitatively similar to the above.

3.1. Stochastic model (simulation). The above deterministic model can be "made stochastic" in several ways, but the simplest, if not most elegant and complete, is to computer-simulate: (a) discretize time in equal-sized steps; (b) allow a discrete-time-step version of (2.1) to define the mean of a Markov stochastic process with (c) normal/Gaussian increments whose mean(s) are defined as in (b) and with variances equal to (or proportional to) the above incremental means, as would be appropriate for a diffusion approximation of a simple birth-death process. The latter may be more appropriate for adducts than for chemical concentration or for the enzyme. Formalize as follows, making time steps unity on an appropriate scale.

Mean sequence.

$$
\begin{gathered}
A(s+1)=A(s)+\lambda_{0}+\lambda_{1} T(s)-\mu_{R A} R(s) A(s)-\delta_{A M} A(s) \\
\equiv A(s)+\Delta A(s) \\
T(s+1)=T(s)+\tau_{C}(s)-\delta_{T} T(s)-\mu_{R T} R(s) T(s) \\
\equiv T(s)+\Delta T(s) \\
R(s+1)=R(s)+\beta(\bar{R}-R(s))-\delta_{R} R(s) \\
-\mu_{R A} R(s) A(s)-\mu_{R T} R(s) T(s) \\
=R(s)+\Delta R(s) .
\end{gathered}
$$


Stochastic increments.

$$
\begin{aligned}
\Delta \mathbf{A}(s)= & \lambda_{0}+\sigma_{0}(s) \Delta \mathbf{W}_{0}(s)+\lambda_{1} T(s)+\sigma_{1}(s) \Delta \mathbf{W}_{1}(s)-\mu_{R A} R(s) A(s) \\
& -\sigma_{R A}(s) \Delta \mathbf{W}_{R A}(s)-\delta_{A M} A(s)-\sigma_{A M}(s) \Delta \mathbf{W}(s)
\end{aligned}
$$

where

$$
\begin{aligned}
\sigma_{0}^{2}(s) & =\xi_{0} \lambda_{0}, \\
\sigma_{1}^{2}(s) & =\xi_{1} \lambda_{1} T(s), \\
\sigma_{R A}^{2} & =\xi_{R A} \mu_{R A} R(s) A(s), \\
\sigma_{A M}^{2}(s) & =\xi_{A M} \delta_{A M} A(s) .
\end{aligned}
$$

The terms $\Delta W_{i}$ are mutually independent and normal/Gaussian with mean 0 and variance 1 (if a different time step, the variance $=$ time step). The constants $\xi_{0}, \xi_{1}, \xi_{R A}$ and $\xi_{A M}$, are introduced so as to allow variability adjustment; putting them all equal to unity simulates a diffusion approximation to a simple birth-death model.

Likewise,

$$
\begin{gathered}
\Delta \mathrm{T}(s)=\tau_{C}(s)+\sigma_{C}(s) \Delta \mathbf{W}_{C}(s)-\delta_{T} T(s)-\sigma_{T}(s) \Delta \mathbf{W}_{T}(s) \\
-\mu_{R T} R(s) T(s)-\sigma_{R T}(s) \Delta \mathbf{W}_{R T}(s), \\
\sigma_{C}^{2}(s)=\xi_{C} \tau_{C}(s) \\
\sigma_{T}^{2}(s)=\xi_{T} \delta_{T} T(s) \\
\sigma_{R T}^{2}(s)=\xi_{R T} \mu_{R T} R(s) T(s) .
\end{gathered}
$$

Finally

$$
\begin{aligned}
\Delta \mathbf{R}(s)= & \beta(\bar{R}-R(s))+\sigma_{\beta}(s) \Delta \mathbf{W}_{\beta}(s)-\delta_{R} R(s)-\sigma_{R}(s) \Delta \mathbf{W}_{R}(s) \\
& -\mu_{R A} R(s) A(s)-\sigma_{R A}(s) \Delta \mathbf{W}_{R A}(s) \\
& -\mu_{R T} R(s) T(s)-\sigma_{R T}(s) \Delta \mathbf{W}_{R T}(s) .
\end{aligned}
$$

It should be clear how to write down formulas for $\sigma_{\beta}^{2}$ and so forth. Form the simulation realization or single-sample history of mutual state component evolution as follows:

$$
\begin{aligned}
& \mathbf{A}(s+1)=\mathbf{A}(s)+\Delta \mathbf{A}(s), \\
& \mathbf{T}(s+1)=\mathbf{T}(s)+\Delta \mathbf{T}(s), \\
& \mathbf{R}(s+1)=\mathbf{R}(s)+\Delta \mathbf{R}(s) .
\end{aligned}
$$


Start from initial conditions. Slight modifications will be necessary near boundaries to retain $\mathbf{R}(s)$ values positive and never greater than $\bar{R}$; also $\mathbf{T}(s)$ and $\mathbf{A}(s)$ are positive for all $s$. Note that there is a normal/Gaussian Wiener process increment with each incremental component; some are common to two state-variable increments, e.g. $\Delta \mathbf{W}_{R T}$ is in common with $\Delta \mathbf{T}(s)$ and $\Delta \mathbf{R}(s)$. The increment components that are not common to others can be combined so as to make necessary generation of just one (independent) increment component. The formulation of (3.2)-(3.12) offers many possibilities to introduce stochastic noise into the deterministic model. Different model behavior can result from different models of stochastic noise. Simulations with small noise $\xi=0.001$ display the behavior that the variables vary about the stable point at which the simulation is initiated.

Mathematical theory. Features of the escape from the neighborhood of either of two possible local stability points can be treated mathematically. This topic is called the exit problem and it is related to the theory of large deviations. Prominent contributors are Schuss (1980), Varadhan (1984), Aldous (1989), Simonian (1995) and Freidlin and Wentzell (1984). An accessible textbook is Bucklew (1990). Attempts to mathematically calculate features of the process of jumping between stable points are not made in this paper; the qualitative features of the process are illustrated by simulation results.

Example 1. Figs. 3-8 illustrate hypothetical time developments of adduct populations and corresponding amounts of enzyme. The initial values of $A$, $R$ and $T$ are set equal to the largest root $R_{U}$ of the cubic equation equivalent to (3.1) and the corresponding values of $A$ and $T$; the $\xi_{C}=25$ and the other $\xi_{i}=1$. Two replications are displayed in Figs. 3-5; both depend on the same parameters, but flare-ups of adducts appear at quite different times. These high-adduct periods correspond to jumps from $R$ values near $R_{U}$ to others near $R_{L}$ (and in the present case rather quickly back), which are triggered by high fluctuations in internal toxin $\mathbf{T}(t)$ caused initially by random fluctuations in $\tau(t)$, the amount of toxic chemical entering the cell. Figure 6 displays results for one replication. Figures 7-9 display time series of the amount of enzyme, the simulated numbers of adducts and the amount of toxin along with histograms of the values. The histogram of the amount of enzyme has the most pronounced bimodal appearance. The less pronounced apparent bimodality in the histograms of numbers of adducts and the amounts of toxin presumably reflects the fact that the derivatives of these two quantities are functions of the amount of enzyme, and so the number of adducts and the amount of toxin tend to be more related to the area under the enzyme time series and are thus smoothed. In these figures, all based on hypothetical parametric values, bistability is plainly visible. However, the jumps are not as extreme as those 


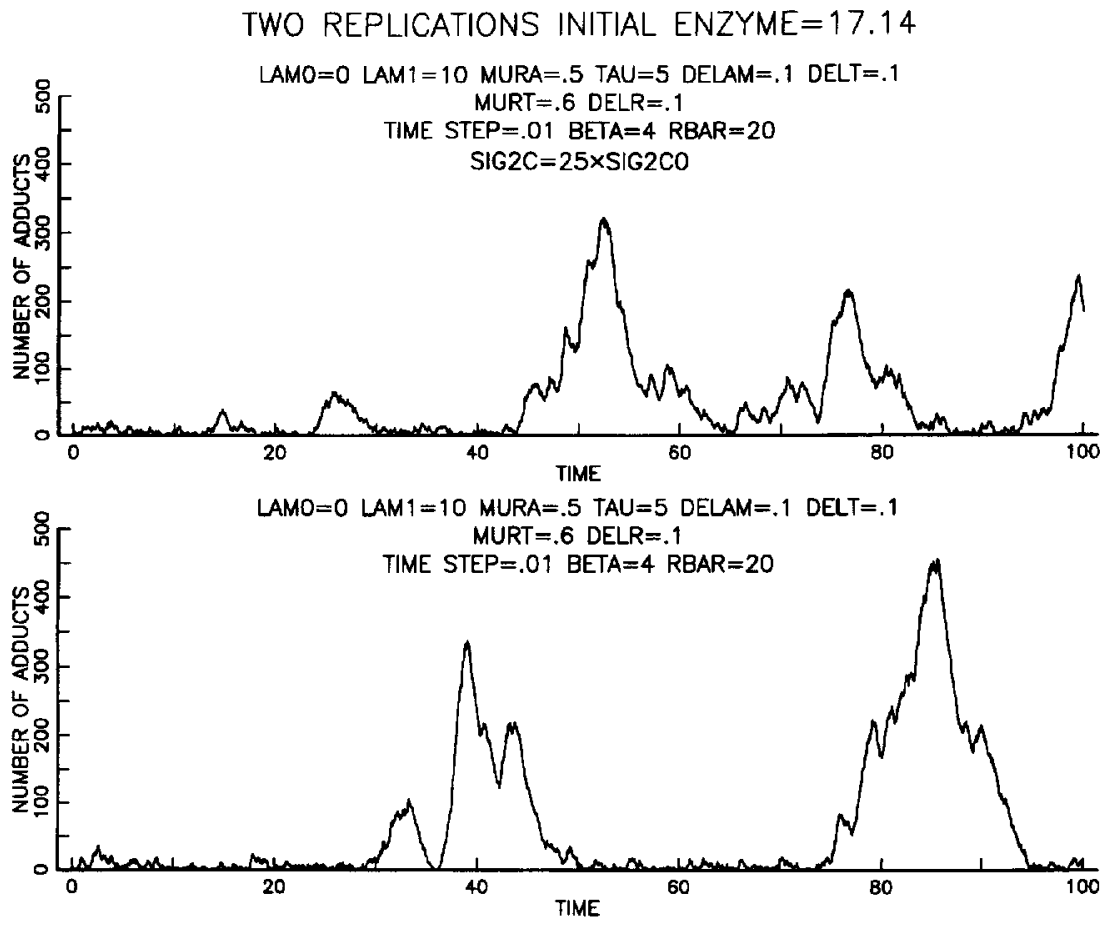

Figure 3. Two replications of time development of number of adducts.

suggested by the deterministic model. The stochastic model introduces an apparent damping effect on the number of adducts that is not predicted by the deterministic model. A burst of adducts can be expected to precede and trigger other events upon successive replication after fixation of single or multiple mutations. Another possibility, also not currently modeled, is the possibility of actual cell death through necrosis or apoptosis when the intracellular toxin is relatively high and adducts are present.

Example 2. Dose-response simulation: Enzyme and adduct distributional summaries depending on the mean toxin input rate $(\tau)$ and noise level. In toxicological terms the toxic chemical input to the cell $\tau$ is a dose and the number of adducts $\mathbf{A}(t)$ is a response. A related, more meaningful response could be cancerous material that resulted from the adducts, but the adducts themselves will suffice. Simulations of our model are suggestive about the shape of a dose-response relationship.

To illustrate the dose-response relationships the following parameterization of the model has been simulated: $\lambda_{0}=0, \lambda_{1}=10, \mu_{R A}=0.5, \delta_{A M}=0.5$, $\delta_{T}=0.5, \mu_{R T}=0.6, \delta_{R}=1, \beta=4$, and $\bar{R}=20$. We have let time run for 100 units with time steps of 0.005 . Only one sample function or history has been generated for each case because of time limitations. Furthermore, we have considered various levels of endogenous and exogenous (toxin input) 
TWO REPLICATIONS INITIAL ENZYME $=17.14$
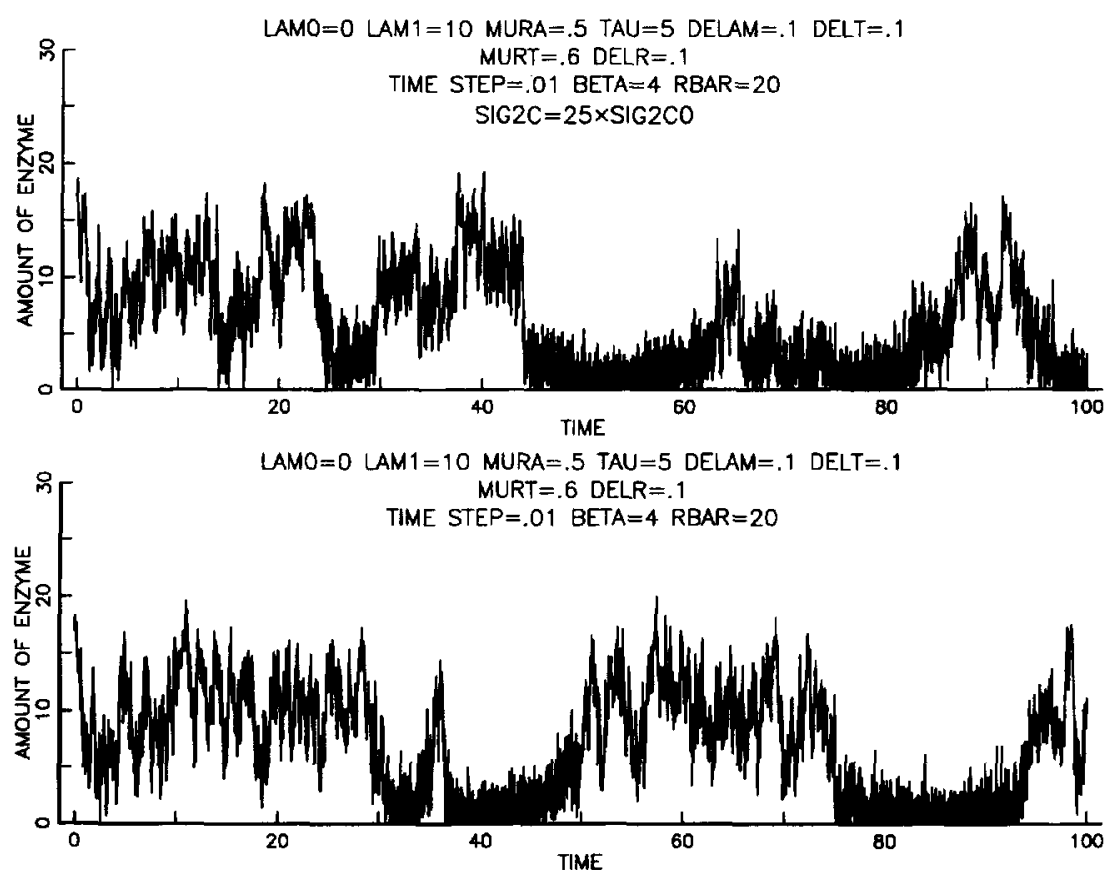

Figure 4. Two replications of time development of amount of enzyme.

TWO REPLICATIONS INITIAL ENZYME $=17.14$
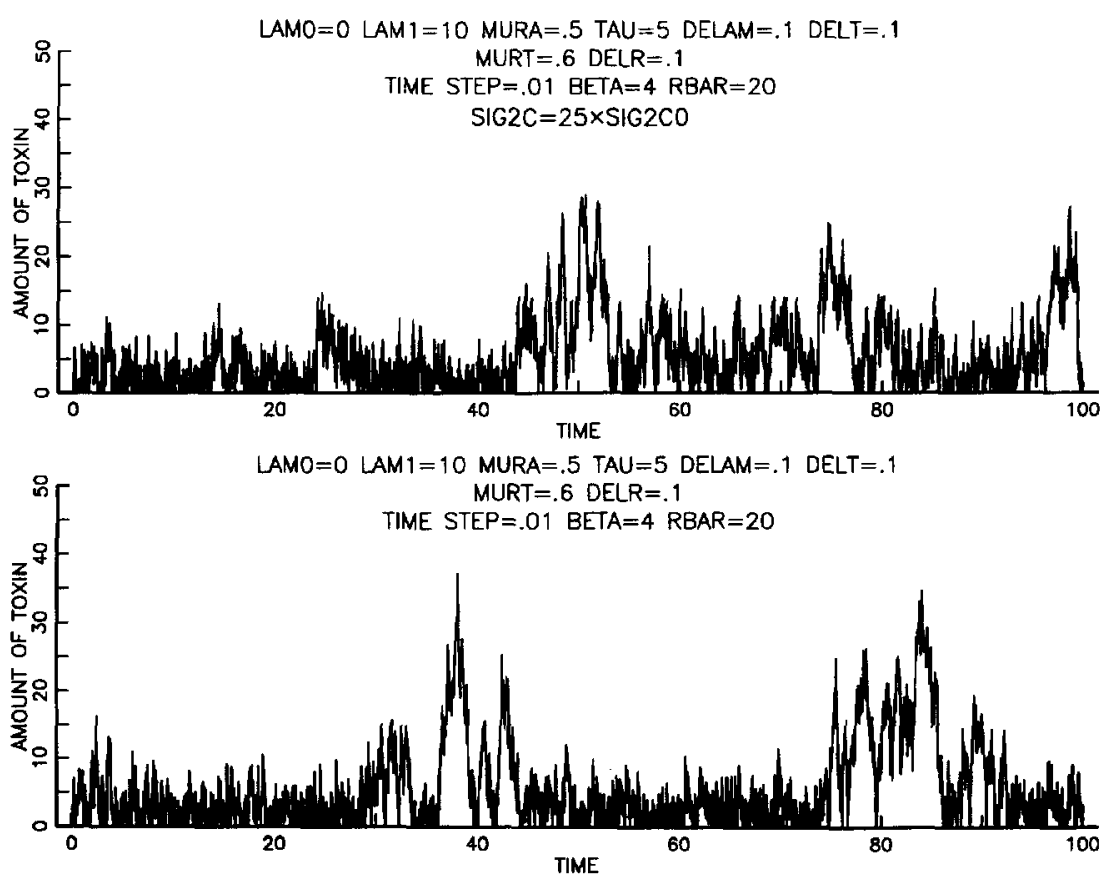

Figure 5. Two replications of time development of amount of toxin. 
INITIAL ENZYME $=17.14$
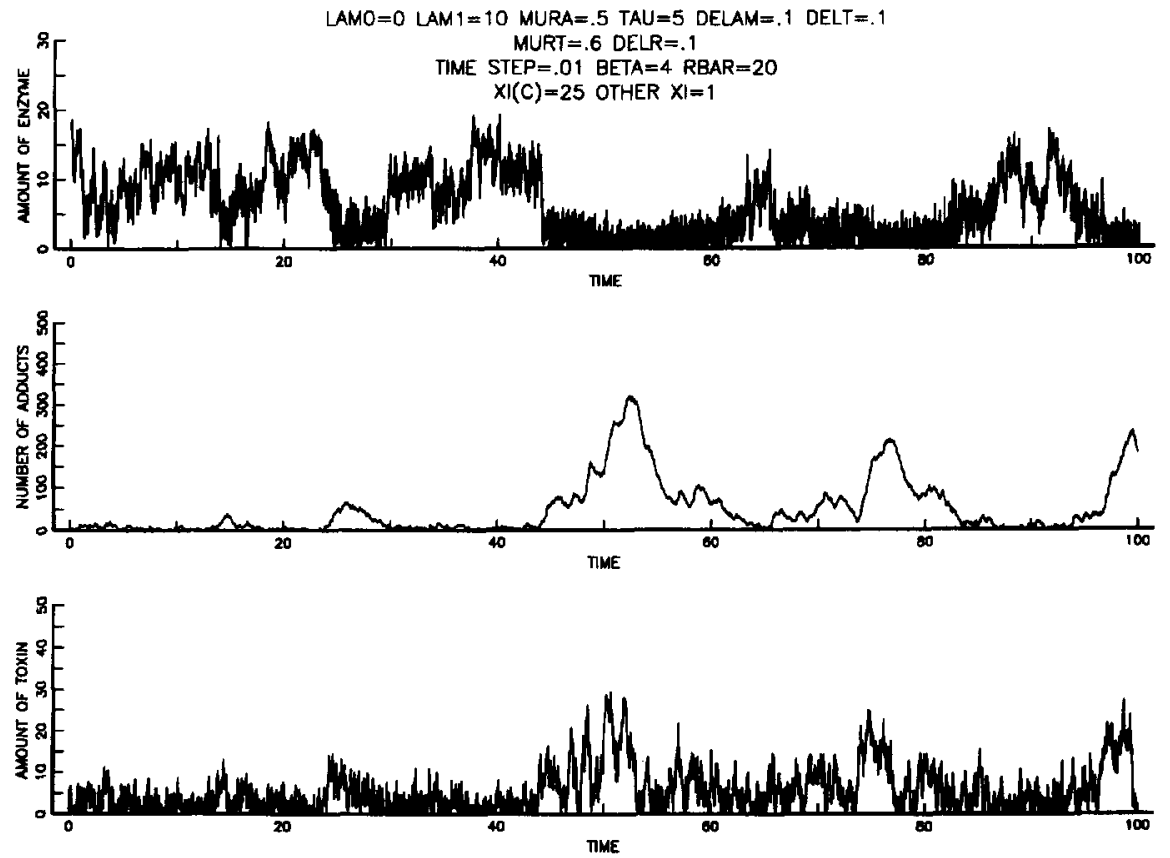

Figure 6. One replication of time development of number of adducts and amounts of enzyme and toxin.

REPLICATION INITIAL ENZYME $=17.14$
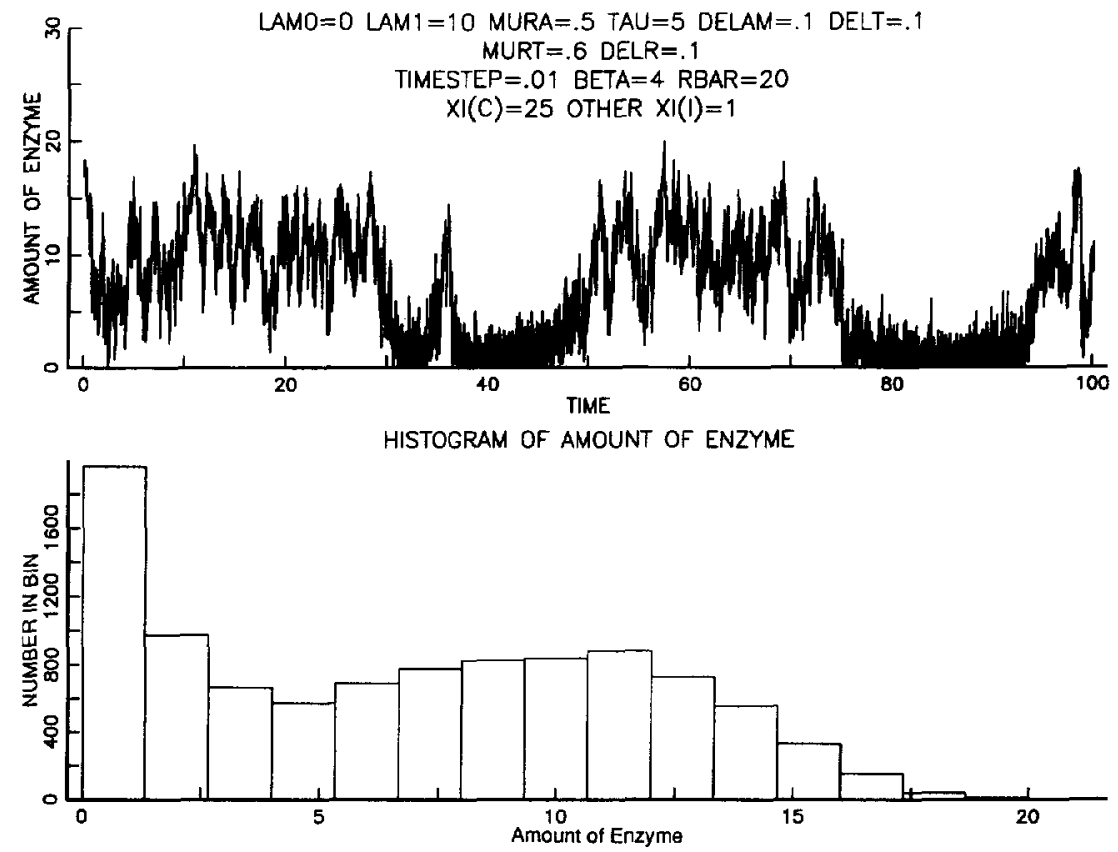

Figure 7. One replication of time development of amount of enzyme. 


\section{REPLICATION INITIAL ENZYME $=17.14$}
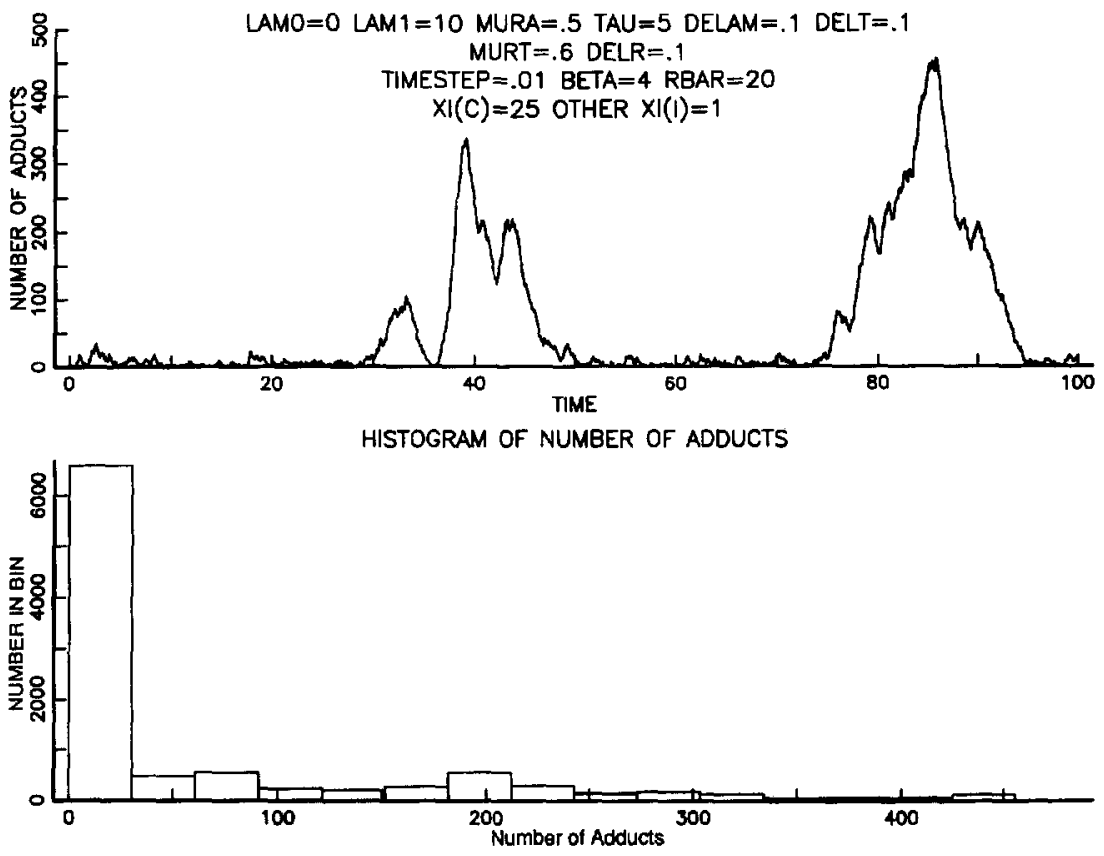

Figure 8. One replication of time development of number of adducts.
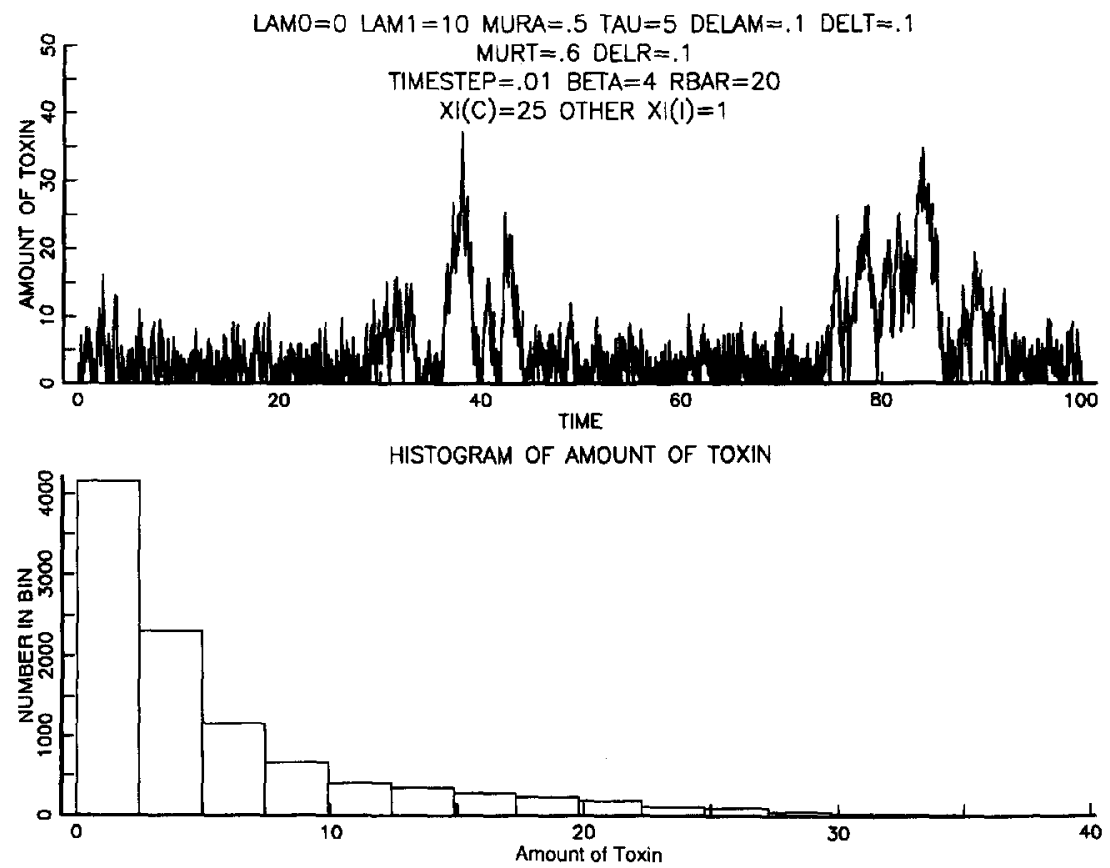

Figure 9. One replication of time development of amount of toxin. 

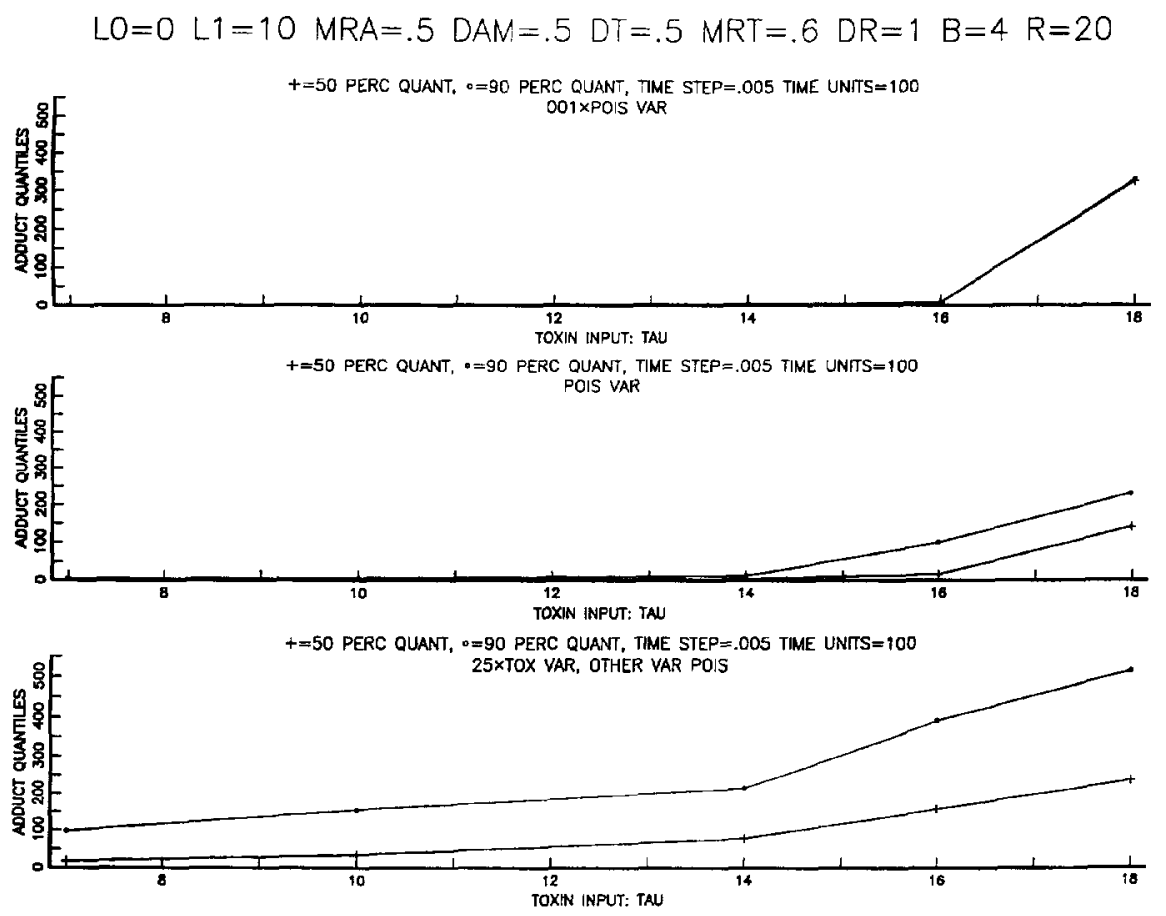

Figure 10. Quantiles of number of adducts for a replication for various toxin inputs and noise variances.

noise: smallest being Poisson with $\xi_{i}=0.001$ in (3.6); second smallest being Poisson $\xi_{i}=1$; largest also Poisson, $\xi_{i}=1.0$ except $\xi_{T}=25$. We have summarized the responses (e.g. total adducts present each time step) by the median (50\% point) and 0.9 quantile (90\% point) over each case $=$ sample history. The results are displayed in the graphs of Figs. 10 and 11.

The graphs indicate the possibility of a sharp non-linearity of threshold effect; this possibility is supported by examination of (3.1a) and particularly Fig. $2 \mathrm{a}-\mathrm{c}$ : As $\tau_{C}$, the mean toxin input, is increased, the solid curves of Fig. 2 tend to rise vertically. At some point Fig. 2a becomes like Fig. 2b, with the sudden appearance of a small-enzyme-concentration stable point. This corresponds at first to an occasional high-adduct presence, but as mean toxin input is increased further the enzyme repair becomes saturated and the adduct population grows rapidly, as indicated by both median and $90 \%$ point. Furthermore, there is some tendency for relatively high variability of toxin $\tau$ to produce even greater adduct accumulation or more pronounced threshold effect.

The above example is the consequence of a simple, but biologically plausible model for a single cell. It does not necessarily imply that all cells in an organ (or on a dish in in vitro studies) will exhibit threshold effects. First, cells may well differ in their individual enzyme-protective capabilities: 

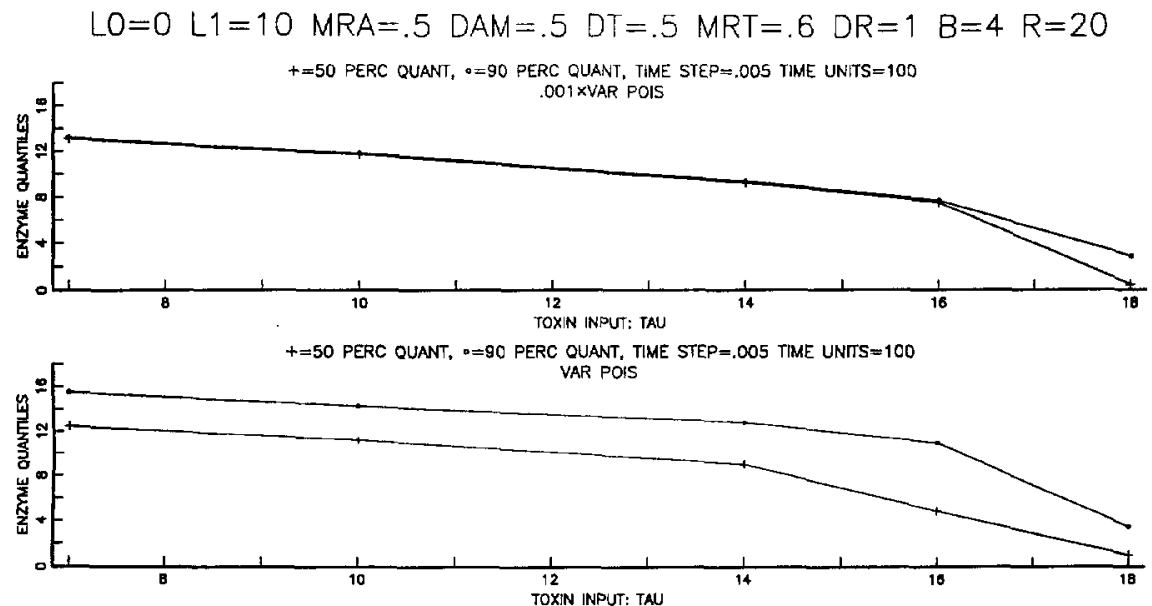

$+=50$ PERC QUANT, $=90$ PERC QUANT, TIME STEP $=.005$ TIME UNITS $=100$

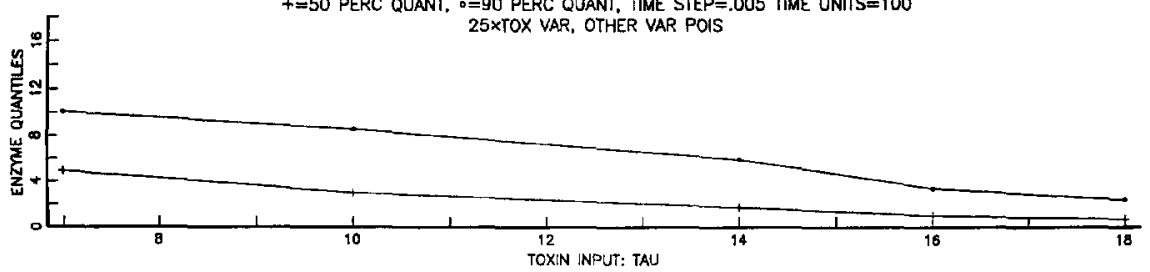

Figure 11. Quantiles of amount of enzyme for a replication for various toxin inputs and noise variances.

some may have relatively high values of $\bar{R}, \beta$, which give them an advantage over others. Second, the model neglects any signaling between cells and consequently protective effects of apoptosis or necrosis.

4. Discussion. DNA abnormalities, e.g. adducts related to chemical exposure, are linked to mutation and the process of carcinogenesis. Hence their existence and relative prevalence within a tissue or cell may be useful as a risk analysis tool. However, the number of DNA adducts within any given cell of an organism on a temporal scale after acute or chronic exposures may be highly variable as a function of many interrelated biological processes.

In the present non-linear dynamic model the possible effect of initial conditions is dramatically illustrated: even a sudden brief shift of intracellular toxic chemical concentration can initiate a qualitative shift in cell condition, both inducing adduct formation and hobbling the cell's repair mechanism by binding the repair enzyme. The biological significance of the model is that such shifts, occurring within a context of replication across a number of cells, can lead to adduct fixation as mutation and preconditions for transformation depending on the site, penetrance and expressivity of the event. The effect may be selective for individuals in the sense that some may have more resistance to assault owing to greater repair capability: 
larger enzyme production attributable to relatively larger propensity for enzyme creation in (2.3), leading to a greater-than-average repair enzyme concentration. Alternatively, there could exist a greater selectivity in binding of the repair enzyme to DNA adducts rather than toxin, i.e. $\mu_{R A}>\mu_{R T}$ in (2.2) and (2.3) in some individual or tissues, thus leading to greater resistance to toxin. On the other hand, if propensity to form adducts is characteristic of at least some cells, i.e. reflected in relatively large parameters $\lambda_{0}$ and $\lambda_{1}$, and perhaps also in outsized fixation rate $\delta_{A M}$ (see (2.1)), then there can be interindividual variations that could quantitatively describe an inherited predisposition to cancer as discussed by Sommerfeld et al. (1995). This characteristic would be independent of the many preexisting mutations in genes known to be involved in the appearance of cancer in certain individuals.

Experimental methods do not now exist to adequately deal with the complexities of rapid shifts in equilibrium affecting DNA adduction or mutation in complex organisms or tissues composed of many different cell types. However, the reported model does suggest that it is relevant to develop analytical approaches to address these issues in the future based on cultured cells with various well-defined initial and induced levels of repair and detoxifying enzymes. Such studies might enhance the capability to predict the more specific responses of certain individuals to exposure.

\section{APPENDIX}

Cubic Equation for Enzyme Fixed Points. The cubic equation that describes the fixed points of (2.1)-(2.3), specifically the solutions of (3.1), is

$$
\begin{aligned}
(\beta+ & \left.\delta_{R}\right) \mu_{R A} \mu_{R T} R^{3}+\left[-\beta \bar{R} \mu_{R A} \mu_{R T}+\left(\beta+\delta_{R}\right)\left(\mu_{R A} \delta_{T}+\mu_{R T} \delta_{A M}\right)+\lambda_{0} \mu_{R A} \mu_{R T}\right. \\
& \left.+\tau_{C} \mu_{R T} \mu_{R A}\right] R^{2}+\left[-\beta \bar{R}\left(\mu_{R A} \delta_{T}+\mu_{R T} \delta_{A M}\right)+\left(\beta+\delta_{R}\right) \delta_{A M} \delta_{T}\right. \\
& \left.+\lambda_{0} \mu_{R A} \delta_{T}+\lambda_{1} \tau_{C} \mu_{R A}+\tau_{C} \mu_{R T} \delta_{A M}\right] R-\beta \bar{R} \delta_{A M} \delta_{T}=0 .
\end{aligned}
$$

Division by $\left(\beta+\delta_{R}\right) \mu_{R A} \mu_{R T}$ puts this into the form in Hodgman (1963) or Press et al. (1992):

where

$$
x^{3}+a x^{2}+b x+c=0,
$$

$$
\begin{aligned}
& a=\frac{\left[-\beta \bar{R} \mu_{R A} \mu_{R T}+\left(\beta+\delta_{R}\right)\left(\mu_{R A} \delta_{T}+\mu_{R T} \delta_{A M}\right)+\left(\lambda_{0}+\tau_{C}\right) \mu_{R A} \mu_{R T}\right]}{\left(\beta+\delta_{R}\right) \mu_{R A} \mu_{R T}}, \\
& b=\frac{\left[-\beta \bar{R}\left(\mu_{R A} \delta_{T}+\mu_{R T} \delta_{A M}\right)+\left(\beta+\delta_{R}\right) \delta_{A M} \delta_{T}+\lambda_{0} \mu_{R A} \delta_{T}+\lambda_{1} \tau_{C} \mu_{R A}+\tau_{C} \mu_{R T} \delta_{A M}\right]}{\left(\beta+\delta_{R}\right) \mu_{R A} \mu_{R T}}, \\
& c=\frac{-\beta \bar{R} \delta_{A M} \delta_{T}}{\left(\beta+\delta_{R}\right) \mu_{R A} \mu_{R T}} .
\end{aligned}
$$


Next, put

$$
Q=\frac{a^{2}-3 b}{9}, \quad S=\frac{2 a^{3}-9 a b+27 c}{54} .
$$

If $S^{2}<Q^{3}$, there are three real roots; if $S^{2}>Q^{3}$, there is one real root (and two imaginaries); if $S^{2}=Q^{3}$, there will be equal roots.

No attempt has been made to simplify or interpret the formidable algebraic expressions that are obtained from the above formal manipulations. Note, however, that if there is no toxin input, i.e. $\tau_{C}(t) \equiv 0$, then $T(t)=0$ and the cubic (A.1) reduces to the quadratic

$$
\left(\beta+\delta_{R}\right) \mu_{R A} R^{2}+\left[-\beta \bar{R} \mu_{R A}+\left(\beta+\delta_{R}\right) \delta_{A M}+\lambda_{0} \mu_{R A}\right] R-\beta \bar{R} \delta_{A M}=0
$$

It is apparent from the explicit solution that there will always be one positive fixed point, which will be the largest solution of (A.5). This will be a stable point. This conclusion follows directly from a graphical argument like that used before.

\section{REFERENCE}

Alberts, B., D. Bray, J. Lewis, M. Ratt, K. Roberts and J. D. Watson. 1994. Molecular Biology of the Cell, New York: Garland.

Aldous, D. 1989. Probability Approximations via the Poisson Clumping Heuristic. New York: Springer-Verlag.

Beltrami, E. 1987. Mathematics for Dynamic Modeling. New York: Academic Press.

Board on Environmental Studies and Toxicology. Commission on Life Sciences. National Research Council. 1989. Biologic significance of DNA adducts and protein adducts. In Drinking Water and Health. Selected Issues in Risk Assessment, Vol. 9, pp. 6-59. Washington, DC: National Academy Press.

Bronstein, S. M., J. E. Cochrane, T. R. Craft, J. A. Swenberg and T. R. Skopek. 1991. Toxicity, mutagenicity, and mutational spectra of $N$-ethyl- $N$-nitrosourea in human cell lines with different DNA repair phenotypes. Cancer Res. 51, 5188-5197.

Bronstein, S. M., T. R. Skopek and J. A. Swenberg. 1992. Efficient repair of $O^{6}$-ethylguanine, but not $O^{4}$-ethylthymine or $O^{2}$-ethylthymine, is dependent upon $O^{6}$-alkylguanine-DNA alkyltransferase and nucleotide excision repair activities in human cells. Cancer Res. 52, 2008-2011.

Bucklew, J. A. 1990. Large Deviation Techniques in Decision, Simulation, and Estimation. New York: Wiley.

Burkhart, J. G. 1995. Perspectives on molecular assays for measuring mutation in humans and rodents. Environ. Mol. Mutagen. 25, 88-101.

Chae, M. Y., M. G. McDougall, M. E. Dolan, K. Swenn, A. E. Pegg and R. C. Moschel. 1994. Substituted $O^{6}$-benzylguanine derivatives and their inactivation of human $O^{6}$-alkylguanine-DNA alkyltransferase. J. Med. Chem. 37, 342-347.

Fox, M. and G. P. Margison. 1988. Expression of an E. coli $O^{6}$-alkylguanine DNA alkyltransferase gene in Chinese hamster cells protects against $N$-methyl and $N$-ethylnitrosourea induced reverse mutation at the hypoxanthine phosphoribosyl transferase locus. Mutagenesis 3, 409-413.

Freidlin, M. I. and A. D. Wentzell. 1984. Random Perturbations of Dynamical Systems. New York: Springer-Verlag.

Hodgman, C. D. (Ed). 1963. CRC Standard Mathematical Tables, 12th ed. Cleveland, OH: Chemical Rubber Publishing Company.

Hora, J. F., A. Eastman and E. Bresnick. 1983. $O^{6}$-methyl guanine methyltransferase rat liver. Biochemistry 22, 3759-3763.

Lijinsky, W., A. E. Pegg, M. R. Anver and R. C. Moschel. 1994. Effects of inhibition of $O^{6}$-alkylguanine-DNA alkyltransferase in rats on carcinogenesis by methylnitrosourea and ethylnitrosourea. Japan J. Cancer Res. 85, 226-230. 
Michaelson, J. 1993. Biology of disease, cellular selection in the genesis of multicellular organization. Laboratory Investigation 69, 136-152.

Mineura, K., I. Izumi, K. Watanabe, M. Kowada, K. Kohda, K. Koyama, I. Terashima and M. Ikenaga. 1994. Enhancing effect of $O^{6}$-alkylguanine derivatives on chlorethylnitrosourea cytotoxicity toward tumor cells. Int. J. Cancer 58, 706-712.

Pegg, A. E. 1990. Mammalian $O^{6}$-alkylguanine-DNA alkyltransferase: regulation and importance in response to alkylating carcinogenic and therapeutic agents. Cancer Res. 50, $6119-6129$.

Pegg, A. E. and T. L. Byers. 1992. Repair of DNA containing $O^{6}$-alkylguanine. FASEB J. 6, 2302-2310.

Press, W. H., S. A. Teukolsky, W. T. Vetterling and B. P. Flannery. 1992. Numerical Recipes in Fortran, 2nd. ed. Cambridge, UK: Cambridge University Press.

Schuss, Z. 1980. Theory and Applications of Stochastic Differential Equations. New York: Wiley.

Simonian, A. 1995. Asymptotic distribution of exit time for small-noise diffusions. SIAMJ. Appl. Math. 55, 809-826.

Singer, B. 1985. In vivo formation and persistence of modified nucleosides resulting from alkylating agents. Environ. Health Persp. 62, 41-48.

Sommerfeld, H. J., A. K. Meeker, E. M. Posadas and D. S. Coffey. 1995. Frontiers in prostate cancer. Cancer Suppl. 75, 2027-2035.

Strogatz, S. H. 1994. Nonlinear Dynamics and Chaos. Reading, MA: Addison-Wesley.

Varadhan, S. 1984. Large Deviations and Applications. Philadelphia, PA: SIAM.

Received 21 September 1995

Revised version accepted 16 June 1996 